\title{
Primary Lymphoma of the Thyroid
}

\author{
Jee Sook Hahn', Hyun Cheol Chung', Yoo Hong Min', Yun Woong Ko \\ Cheong Soo Park ${ }^{2}$, Chang Ok Suh ${ }^{3}$, Gwi Eon Kim ${ }^{3}$ and Woo Ick Yang
}

\begin{abstract}
Primary lymphoma presenting in the thyroid gland is uncommon. A review of the Yonsei University Medical Center experience between 1982 and 1994 was performed retrospectively to assess the treatment outcome and prognostic factors. There were four females and one male, and the median age was 65 years. All 5 cases presented with a neck mass. Two of them had co-existing biopsyproved Hashimoto's thyroiditis and three cases were each in a hypothyroid state. All cases with nonHodgkin's disease were of intermediate grade. One case was in stage IE and four were in stage IIE. Three cases were treated with surgery alone and two cases with bulky inoperable stage IIB were treated with chemo-radiotherapy. Chemotherapy induced a complete response in one and a partial response in the other with minimal transient toxicity. As the questions regarding justification for extensive surgical intervention increase, combined chemo-radiotherapy can be suggested as an initial treatment even in stage I, and stage II thyroid lymphoma based on prognostic factor evaluation.
\end{abstract}

Key Words: Thyroid, lymphoma, chemo-radiotherapy

Primary thyroid lymphoma is an uncommon disease, comprising $2.5 \%$ of all malignant lymphomas (Freeman et al. 1972; Compagno and Oertel, 1980: Tupchong et al. 1986), and approximately $5 \%$ of thyroid malignant neoplasms (Staunton and Greening, 1973). In contrast to other types of non-Hodgkin's lymphomas(NHL), it is more common in elderly female cases (Burke et al. 1979; Grimley and Oates, 1980). The special features of this disease include the association with Hashimoto's thyroiditis, the frequency of isolated gastrointestinal relapse (Goudie and Angouridakis, 1970; Hamburger et al. 1983; Tsang et al. 1993) and controversy regarding the extent of surgical management.

The conventional approach to treatment of this disease has been to combine surgical

Received March 27, 1995

Accepted August 17, 1995

Departments of Internal Medicine', General Surgery', Radiation Oncology ${ }^{3}$ and Rathology4, Yonsei University College of Medicine, Seoul, Korea

Address reprint requests to Dr. JS Hahn, Department of Internal Medicine, Yonsei University College of Medicine, C.P.O. box 8044, Seoul 120-752, Korea resection and post-operative radiotherapy, which has induced a $30 \sim 70 \%$ overall survival rate(Staunton and Greening, 1976; Kapadia et al. 1982; Tennvall et al. 1987). However, with the development of multi-agent chemotherapy, combined chemo-radiotherapy has become more and more popular as initial therapy (Vigliotti et al. 1986; Skarsgard et al. 1991). This has brought into question the role of surgery as a primary treatment (Rosen et al. 1988).

Reviewing the clinical and laboratory features of our cases, we report our experiences, emphasizing early, and correct diagnosis and conservative evaluation of the optimal treatment for thyroid lymphoma.

\section{MATERIALS AND METHODS}

Five cases with primary thyroid lymphoma at the Yonsei University Medical Center (YUMC) were reviewed over a 13-year period between 1982 to 1994 . Four cases were female and 1 was male, with a median age of 65 years (range: 52 to 72 years). The original bi- 
opsy materials were re-evaluated and classified by NCI working formulation. Staging was done according to the Ann Arbor Criteria (Carbone et al. 1971). Staging work-up included history, physical examination, blood chemistry, chest X-ray, computed tomography, gallium scan, bone scan, bone marrow aspiration and biopsy.

A diagnostic lymph node biopsy or surgical intervention was performed in each of the five cases. Surgery entailed either a lobectomy or a subtotal-total thyroidectomy. Radiotherapy with $4,000 \sim 6,500$ cGy tumor dosage was delivered using megavoltage equipment, encompassing the thyroid, cervical lymph nodes, supraclavicular fossa, and mediastinum with or without axillary fossa. Chemotherapy regimens consisted of cyclophosphamide, adriamycin, vincristine and prednisolone (CHOP) or cyclophosphamide, vincrinstine and prednisolone (CVP). Survival time was calculated from the time of initial treatment to the date of death or most recent follow-up date.

\section{RESULTS}

\section{Patient characteristics}

A non-tender growing neck mass with a less than seven month (median 2.8 month) history was the presenting symptom in all cases. One case presented as a growing mass with pressure symptoms, such as dyspnea, hoarseness and superior vena cava syndrome, and " $B$ " symptom. Each of three cases presented as an enlarged thyroid gland in the form of a discrete nodule and the other two presented as a diffuse goiter. The median largest diameter of the tumor mass was $7.0 \mathrm{~cm}$ (range $5.5 \sim 11.0$ $\mathrm{cm}$ ), with the masses fixed to adjacent soft tissues in all five cases. The mass was confined to one lobe in three, while diffuse bilobar involvement was found in two. Lymphadenopathy was present in four cases, including four cervical lymphadenopathy, two supraclavicular lympalenopathy, and one axillary lymphadenopathy. Mediastinal involvement was found in two cases (Table 1).

Three cases had a history of goiter and two
Table 1. Clinical fetures

\begin{tabular}{lc}
\hline \hline & $\begin{array}{c}\text { Number of } \\
\text { cases }(\%)(\mathbf{n}=5)\end{array}$ \\
\hline History of goiter & $3(60)$ \\
Presenting symptoms & $1(20)$ \\
dyspnea & $1(20)$ \\
hoarseness & $1(20)$ \\
SVC syndrome & $1(20)$ \\
pain & $1(20)$ \\
B symptoms & $1(20)$ \\
Physical findings & \\
neck mass & $5(100)$ \\
fixation & $5(100)$ \\
mass $>$ 10 cm & $2(40)$ \\
diffuse enlargement & $2(40)$ \\
discrete nodule & $3(60)$ \\
single lobe involvement & $3(60)$ \\
right lobe & $3(60)$ \\
bilobar involvement & $2(40)$ \\
lymphadenopathy & $4(80)$ \\
cervical adenopathy & $4(80)$ \\
supraclavicular adenopathy & $2(40)$ \\
axillary adenopathy & $1(20)$ \\
mediastinal involvement & $2(40)$ \\
\hline
\end{tabular}

SVC: superior vena cava

Table 2. Thyroid function

\begin{tabular}{ll}
\hline Hashimoto's thyroiditis & $2 / 5(40 \%)$ \\
Thyroid function & \\
$\quad$ euthyroid & $2 / 5(40 \%)$ \\
hypothyroid & $3 / 5(60 \%)$ \\
Anti-microsomal antibody positivity & $3 / 4(75 \%)$ \\
Anti-thyroglobulin antibody positivity & $3 / 4(75 \%)$ \\
Thyroid scan & \\
$\quad$ Cold nodules & $5 / 5(100 \%)$ \\
\hline
\end{tabular}

of these had simultaneous Hashimoto's thyroiditis. In thyroid function test, three cases were in various stages of hypothyroidism. Anti-microsomal antibody was positive in $75 \%$ (3/4) and anti-thyroglobulin antibody was positive in $75 \%(3 / 4)$ of the cases. All five cases showed a sizable cold area in thyroid scan (Table 2). Fine needle aspiration biopsy (FNAB) was done in three cases; as an initial diagnostic procedure in two, and after regional 
Table 3. Distribution by histology and stages

\begin{tabular}{lcccc}
\hline \multicolumn{1}{c}{ Stage } & PDLL & DM & DL & Total \\
\hline IE & & & 1 & 1 \\
IIE(non-mediastinal) & & 1 & $1^{*}$ & 2 \\
IIE(mediastinal) & 1 & & $1^{*}$ & 2 \\
\hline$\quad$ Total & 1 & 1 & 3 & 5 \\
\hline
\end{tabular}

*clinical stage

PDLL: poorly differentiated lymphocytic lymphoma

DM: diffuse mixed

$\mathrm{DL}$ : diffuse large

Table 4. Treatment by stage

\begin{tabular}{lcccc}
\hline \hline \multicolumn{1}{c}{ Stage } & Surgery & $\begin{array}{c}\text { Surger } \\
\text { +Radio- } \\
\text { therapy }\end{array}$ & $\begin{array}{c}\text { Chemo } \\
\text { - }\end{array}$ & $\begin{array}{c}\text { thadio- } \\
\text { therapy }\end{array}$ \\
\hline IE & 1 & & & 1 \\
IE & 1 & & $1^{*}$ & 4 \\
$\begin{array}{l}\text { (non-mediastinal) } \\
\text { IIE } \\
\text { (mediastinal) }\end{array}$ & & & & \\
\hline \multicolumn{1}{c}{ Total } & 2 & 1 & 2 & 5 \\
\hline
\end{tabular}

*clinical stage

lymph node biopsy in one. The two initial FNAB results misdiagnosed as chronic inflammation in one patient and as papillary type carcinoma in another case; these were finally confirmed as lymphoma after surgery. Three cases underwent diagnostic/therapeutic partial or total thyroid resection on the impression of thyroid cancer. In the remaining two cases, the final diagnosis was established by open biopsy of enlarged cervical or supraclavicular lymph nodes. All five cases showed intermediate-grade non-Hodgkin's lymphoma (one poorly differentiated lymphocytic type, three diffuse large cell types, one diffuse mixed cell type) (Table3).

\section{Treatment outcome}

There was one case with stage I and four cases with stage II disease. Three cases had thyroidectomy including two cases with bilateral total thyroidectomy. Of three cases after surgery, one case received post-operative radiotherapy and two cases(one had a plan of radiotherapy) had no further treatment. Two cases were initially managed with combined chemo-radiotherapy resulting in one complete response and one partial response (Table 4). During chemo-radiotherapy, transient WHO grade II leukopenia and anemia occurred. Mild esophagitis was occurred in one case during radiotherapy. No fatal complications were encountered from four surgery and two chemoradiotherapy. Among five cases, two were lost to follow-up at two and 31 months, respectively, and one died at 31 months (Table 5).

\section{DISCUSSION}

The usual clinical presentation of thyroid lymphoma is a rapidly growing thyroid mass, often with compressive symptoms of dysphagia, dyspnea, stridor or hoarseness. In concordance with other results (Compagno and Oertel, 1980; Ban et al. 1984; Tupchong et al. 1986), all five cases complained of a growing neck mass. The most common presentation form of thyroid mass was a discrete nodular shape. Approximately two-thirds of the cases in the available literature present with nodular shape while one-third show diffuse goiter (Hamburger et al. 1983; Aozasa et al. 1986). In our study, three cases had nodular and two were of diffuse types. Skarsgard et al. (1991) report $73 \%$ incidence of cervical or mediastinal lymphadenopathy. Similarly, two-thirds of our cases presented initially with lymphadenopathy. Two supraclavicular lymphadenopathy and one axillary lymphadenopathy were also found by Blair et al. (1985).

In $80 \sim 83 \%$ of thyroid lymphoma, Hashimoto' $s$ thyroiditis is associated (Hamburger et al. 1983; Aozasa et al. 1986) and symptoms of hypothyroidism are rare unless there is Hashimoto's thyroiditis. Two reports have documented an increased risk (up to 67 times) of thyroid lymphoma developing in cases with bi- 
Jee Sook Hanh, et al.

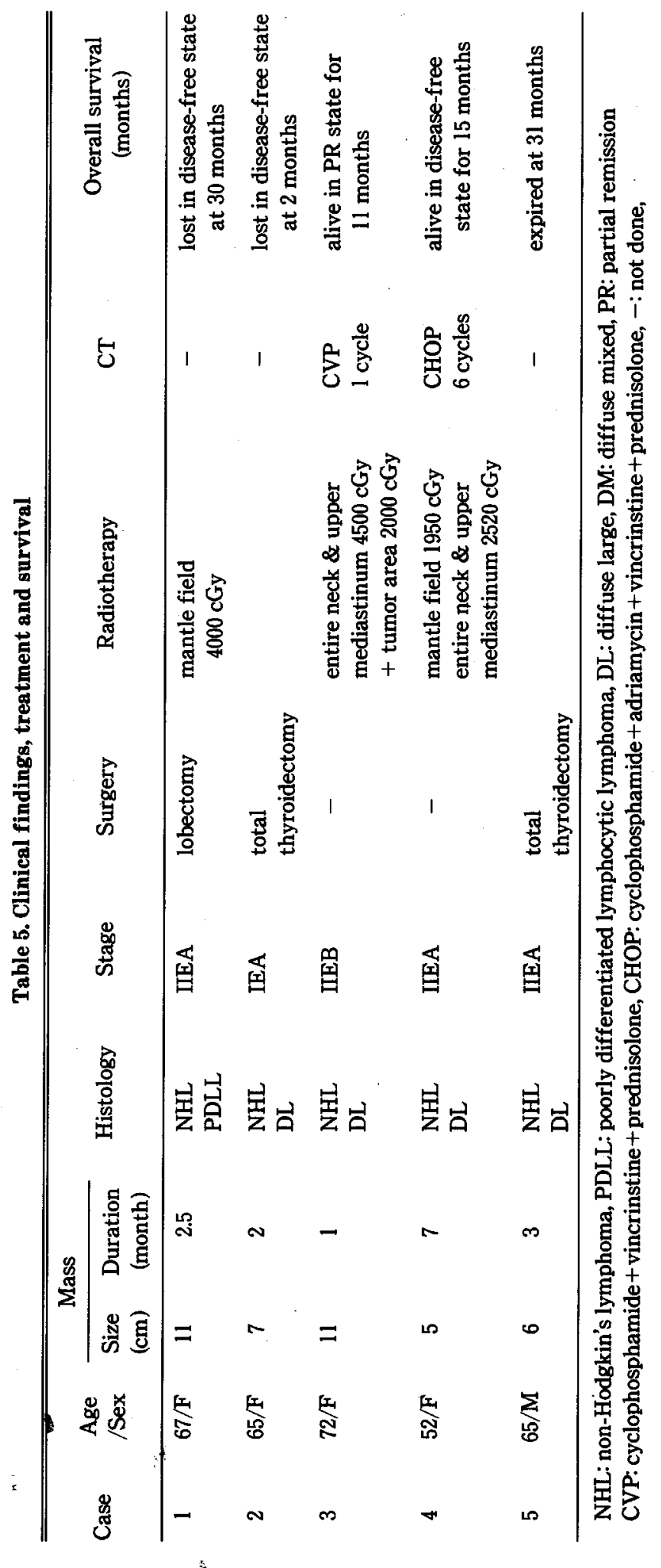


opsy-proved Hashimoto's thyroiditis (Holm et al. 1985; Kato et al. 1985). These frequent findings of co-existance were confirmed in our series; $40 \%$ of our patients had biopsy-proved Hashimoto's thyroiditis and $60 \%$ were in a hypothyroid state. Therefore, if an elderly case presents with a history of Hashimoto's thyroiditis that also has a rapidly growing thyroid nodule, thyroid lymphoma should be suspected. Hamburger et al. (1983) also suggested the following conditions as highly probable co-existing lymphoma in Hashimoto's thyroiditis cases without nodules: cold zone thyroid scan, rapidly growing goiter, enlargement of goiter even with thyroid hormone supplement, and painful, tender goiter with hoarseness.

Even when the diagnostic accuracy of a fine-needle aspiration biopsy(FNAB) increased to around $50 \sim 60 \%$ (Esselstyn and Crile, 1984; Skarsgard et al. 1991), chronic lymphocytic thyroiditis and small cell undifferentiated carcinoma(anaplastic carcinoma) have been confused with thyroid lymphoma (Mambo and Irwin, 1984). In our experience of two cases, the diagnoses were incorrect with FNAB. Taking into account our experience with FNAB, we prefer thyroid biopsy in cases where the clinical impression is of thyroid lymphoma. The rationale of the biopsy is that an incisional thyroid biopsy confirms the histological diagnosis with about a $90 \%$ accuracy rate. Moreover, it supplies a sufficient tissue supply for classification(Skarsgard et al. 1991).

Most of the thyroid lymphomas are of intermediate grade, especially the diffuse large cell type with a frequency rate of $60 \sim 90 \%$ by working formulation (Souhami et al. 1980; Aozasa et al. 1986), and $80 \sim 90 \%$ of this type of lymphoma is in stage I-II (Aozasa et al. 1986; Tsang et al. 1993). Maurer et al. (1979) comment that large non-cleaved cell type predominently shows localized disease, while immunoblastic type and small cleaved cell type usually show advanced disease. Similar results were obtained in our cases and all of our cases were intermediate grade with, stage I-II disease.

The major role of surgery is the resection of operable disease so that minimal disease is present before radiotherapy. As tumors with gross residuals (more than $2.5 \mathrm{~cm}$ ) have failed to achieve permanent local control in $35 \%$, and an additional $23 \%$ showed distant recurrence as a first manifestation of relapse(Rosen et al. 1988), questions have been raised against the justification for extensive surgical intervention other than diagnostic biopsy in bulky stage I-II disease. Even if radiotherapy is combined with surgery, the five-year survival rate is still around $50 \sim 70 \%$ (Kapadia et al. 1982; Tennvall et al. 1987). In our experience of two cases with surgery alone, one died at 31 months, and one was lost to follow-up at two months. One case which received adjuvant radiotherapy with mantle field as Blair et al. (1985) suggested, was disease-free state at 30 month follow-up.

Chemotherapy has been traditionally utilized in stage III and IV disease and in salvage treatment. But a high recurrence rate after local treatment, preponderance of diffuse large cell type, and usage of doxorubicin-based regimens with high complete remission rate $(65 \sim 80 \%)$ have led to the proposal of the use of multi-drug chemotherapy as an initial treatment even in stage I and II with adverse prognostic factors (Tupchong et al. 1986; Leedman et al. 1990; Skarsgard et al. 1991). Leedman et al. (1990) induced complete remission with initial chemotherapy even when the tumor was large and extra-thyroidal invasion was present. Tsang et al. (1993) induced 70\% survival rate with combined chemo-radiotherapy in most of stage I-II cases and did radiotherapy alone only for a small group of stage I cases with a small tumor bulk. Rosen et al. (1988) suggested bulky and/or inoperable tumors may be more effectively managed with a combined regimen including chemoradiotherapy. In our experience, one bulky inoperable stage IIE case received six cycles of CHOP chemotherapy which induced a complete tumor response. Another huge inoperable stage IIE case received one cycle of CVP regimen and radiotherapy which induced partial response. Our experience suggested the possible role of combined chemo-radiotherapy in bulky stage I-II thyroid lymphoma. Both patients with a chemotherapy regimen were tolerable with minor complications such as transient anemia and neutropenia. 
Several studies demonstrate tumor bulk (> $10 \mathrm{~cm}$ ) as the single most important prognostic factor in localized thyroid lymphoma (Rosen et al. 1988; Skarsgard et al. 1991; Tsang et al. 1993) due to local invasion and non-resectability. In our study, two cases showed huge masses(> $10 \mathrm{~cm}$ ). Several unfavorable prognostic factors also have been recognized; advanced age (generally $>60$ ), signs of extra-thyroidal extension (hoarseness, stridor, dysphagia, dyspnea), tumor stage, mediastinal involvement, short duration (<six months) of thyroid mass, tumor fixation and histology (Aozasa et al. 1986; Tupchong et al. 1986). From earlier reports with the broad heading of histiocytic lympho$\mathrm{ma}$, the histologic type did not predict longterm survival in thyroid lymphoma. However, with international working formulation, high-, intermediate- and low-grade thyroid lymphoma showed $13 \%, 79 \%, 92 \%$ five-year survival rate, respectively (Aozasa et al. 1986). Factors that have been associated with improved survival include intra-thyroidal disease (stage I), complete tumor resection, and associated Hashimoto's thyroiditis (Tupchong et al. 1986; Vigliotti et al. 1986). One stage IIE case with hoarseness, B symptoms, large size $(11 \mathrm{~cm})$, mediastinal involvement, extra-thyroidal lymphomatous involvement, and no associated-Hashimoto's thyroiditis showed only partial response after chemo-radiotherapy, while the other stage IIEA case with associated Hashimoto's thyroiditis had a complete response and was disease-free state for 15 months.

As the natural course of the thyroid lymphoma is the same as the other extra-nodal non-Hodgkin's lymphoma, our experience suggested that combined chemo-radiotherapy can be used as an initial treatment even when the patient presents with stage I, II with unfavorable prognostic factors. For the final therapeutic strategy in primary thyroid lymphoma, more case accumulating data is needed.

\section{REFERENCES}

Aozasa $\mathrm{K}$, Inoue $\mathrm{A}$, Tajima $\mathrm{K}$, Miyauchi $\mathrm{A}$,
Matsuzuka F, Kuma K: Malignant lymphomas of the thyroid gland: analysis of 79 patients with emphasis on histological prognostic factors. Cancer 58: 100-104, 1986

Ban JM, Park CS, Lee HC, Huh KB: Primary malignant lymphoma of the thyroid gland. Kor $J$ Surgery 27: 288-293, 1984

Blair TJ, Evans RG, Buskirk SJ, Banks PM, Earle JD: Radiotherapeutic management of primary thyroid lymphoma. Int J Radiat Oncol Biol Phys 11: $365-370,1985$

Burke JS, Butler JJ, Fuller LM: Malignant lymphomas of the thyroid: a clinical pathologic study of 35 patients including ultrastructural observations. Cancer 39: 1587-1602, 1979

Carbone PP, Kaplan HS, Musshoff K, Smithers D, Tubiana M: Report of the committee on Hodgkin's disease staging classification. Cancer Res 31: 1860-1861, 1971

Compagno J, Oertel JE: Malignant lymphoma and other lymphoproliferative disorders of the thyroid gland: a clinicopathologic study of 245 cases. Am J Clin Pathol 74: 1-11, 1980

Esselstyn $C B$, Crile G: valuation of various types of needle biopsies of the thyroid. World $J$ Surg 8: 452-457, 1984

Freeman C, Berg JW, Cutler SJ: Occurrence and prognosis of extranodal lymphomas. Cancer 29: 252-260, 1972

Goudie R, Angouridakis CE: Autoimmune thyroiditis associated with malignant lymphoma of the thyroid. J Clin Pathol 23: 377-381, 1970

Grimley RP, Oates GD: The natural history of malignant thyroid lymphomas. $\mathrm{Br} J$ Surg 67: 475477,1980

Hamburger JI, Miller JM, Kini SR: Lymphoma of the thyroid. Ann Intern Med 99:685-693, 1983

Holm L, Blomgren H, Lowhagen T: Cancer risks in patients with chronic lymphocytic thyroiditis. $N$ Engl J Med 312: 601-604, 1985

Kapadia SB, Deker A, Cheng VS: Malignant lymphoma of the thyroid gland: a clinicopathologic study. Head Neck Surg 4: 270-280, 1982

Kato I, Tajima K, Suchi T, Aozasa K, Matsuzuka, F, Kuma K, Tominaga $S$ : Chronic thyroiditis as a risk factor of B-cell lymphoma in the thyroid gland. Jpn J Cancer Res 76: 1085-1090, 1985

Leedman PJ, Sheridan WP, Downey WF, Fox RM, Martin IR: Combination chemotherapy as single modality therapy for stage IE and IIE thyroid lymphoma. Med J Aust 152: 40-43, 1990

Mambo NC, Irwin SM: Anaplastic small cell neoplasms of the thyroid: An immunoperoxidase 
study. Human pathol 15: 55-60, 1984

Maurer R, Taylor CR, Terry R, Lukes RJ: NonHodgkin's lymphomas of the thyroid: A clinicopathological review of 29 cases applying the Lukes-Collins classification and an immunoperoxidase method. Virch Arch Path Anat Histol 383: 293-317, 1979

Rosen IB, Sutcliffe SB, Gospodarowicz MK, Chua $T$, Simpson WJ: The role of surgery in the management of thyroid lymphoma. Surg 104: 1095-1099, 1988

Skarsgard ED, Connors JM, Robins RE: A current analysis of primary lymphoma of the thyroid. Arch Surg 126: 1199-1204, 1991

Souhami L, Simpson WJ, Carruthers JS: Malignant lymphoma of the thyroid gland. Int $J$ Radiat Oncol Biol Phys 6: 1143-1147, 1980

Staunton HD, Greening WP: Clinical diagnosis of thyroid cancer. Brit Med J 4: 532-535, 1973
Tennvall J, Cavallin-Stahl E, Akerman M: Primary localised non-Hodgkin's lymphoma of the thyroid: a retrospective clinicopathological review. Eur J Surg Oncol 13: 297-302, 1987

Tsang RW, Gospodarowicz MK, Sutcliff SB, Sturgeon JFG, Panzarella T, Patterson BJ: NonHodgkin's lymphoma of the thyroid gland: prognostic factors and treatment outcome. Int J Radiat Oncd Biol Phys 27: 599-604, 1993

Tupchong L, Hughes F, Harmer CL: Primary lymphoma of the thyroid: Clinical features, prognostic factors, and results of treatment. Int $J$ Radiat Oncol Biol Phys 12: 1813-1821, 1986

Vigliotti A, Kong JS, Fuller LM, Velasquez WS: Thyroid lymphomas stages IE and IIE: comparative results for radiotherapy only, combination chemotherapy only, and multimodality treatment. Int J Radiat Oncol Bid Phys 12: 1807 1812,1986 